\title{
Services for Knowledge Resource Sharing \& Management in an Open Source Infrastructure for Lifelong Competence Development ${ }^{*}$
}

\author{
Elena Demidova ${ }^{1}$, Philipp Kärger ${ }^{1}$, Daniel Olmedilla ${ }^{1}$, Stefaan Ternier $^{2}$, Erik Duval $^{2}$, \\ Michele Dicerto ${ }^{3}$, Carlos Mendez ${ }^{4}$, Krassen Stefanov ${ }^{5}$ \\ ${ }^{1}$ L3S Research Center, Hannover, Germany,E-mail: \{demidova,kaerger,olmedilla\}@L3S.de \\ ${ }^{2}$ Katholieke Universiteit Leuven, Leuven,Belgium,E-mail: \{stefaan.ternier,erik.duval\}@cs.kuleuven.be \\ ${ }^{3}$ GIUNTI ILABS, Sestri Levante, Italy,E-mail:m.dicerto@giuntilabs.it \\ ${ }^{4}$ Altran SDB, Madrid, Spain, E-mail: cmendez@altransdb.com \\ ${ }^{5}$ Sofia University, Sofia, Bulgaria,E-mail: krassen@fmi.uni-sofia.bg
}

\begin{abstract}
Access to learning information is still restricted due to the lack of technical and semantic interoperability, locking knowledge resources in disconnected islands. This situation does not provide learners with a motivating environment in which to access and share learning information. In order to target emerging needs for lifelong competence development, a flexible and open source environment for management and sharing of knowledge resources must be built on top of an infrastructure that maximizes the amount of information available, therefore integrating centralised repositories and user desktop resources as well as emergent Web 2.0 applications. This paper describes the challenges and requirements that need to be addressed and presents a set of interfaces and our current implementation of the Knowledge Resource Sharing and Management service oriented architecture.
\end{abstract}

\section{Introduction}

Traditionally, instructional learning has covered the needs of people searching for new labour opportunities, be it in the form of improvements or upgrades in current jobs or as a way to be able to apply for new ones. Nowadays, there is a growing need for more flexible and cost-effective solutions for learners in order to provide lifelong competence development. TENCompetence [1] addresses this need and aims at supporting individuals, groups and organizations by establishing the most appropriate technical and organizational infrastructure, using open source, standards-based, sustainable and innovative technology. Such an infrastructure requires a knowledge resource sharing and management middleware as basis to ensure availability of resources as well as the ability to exchange and (re-)use them.
Knowledge resources ${ }^{1}$ are spread over the web, in centralised repositories, web servers and user desktops. On the one hand, centralised and monolithic repositories (on which e.g., Learning Management Systems rely) represent the traditional and oldfashioned approach for resource sharing. On the other hand, completely decentralised networks like P2P allow users to share content without relying on a third party repository, therefore without loosing control over it. This appealing alternative, which is successfully in use (e.g., Edutella [2] and LionShare [3]), allows learners to share e.g., their desktop resources. Lately, a successful integration of these two kinds of information sources has been demonstrated [4]. However, it does not provide other services (e.g., publishing of knowledge resources).

Currently, emergent Web 2.0 applications (e.g., Flickr [5] and YouTube [6]) provide new means for users to share information with a higher level of motivation than the more old-fashioned repository-like and Peer-to-Peer (P2P) networks. A flexible knowledge resource infrastructure must not only allow searching through heterogeneous information sources but must also provide services like resource publishing, information source and user management as well as resource rating. Unfortunately, there is no standard interface that serves this purpose in a way that all information sources could be easily targeted. This lack of interoperability represents a challenging task: building an infrastructure for sharing and management of knowledge resources, that integrates all relevant

\footnotetext{
* The work on this paper has been partially sponsored by the TENCompetence Integrated Project. Contract 027087.

${ }^{1}$ With knowledge resources we mean the containers that store the explicit knowledge for sharing purposes. Examples are learning objects, articles, books, software programs, informal messages, etc.
} 
services and makes them interoperable over heterogeneous information sources.

In order to achieve the integration of models and tools for the creation, storage and exchange of knowledge resources, we initiated collaboration on the development of simple but flexible and powerful interfaces (as it was done for search with SQI [7]) for the services required in this infrastructure (publishing, information source management, user management and resource rating). In addition, we implemented our infrastructure with the first version of these interfaces, making information accessible in order to better support lifelong learning and at the same time enhance the learning experience.

\section{Service Interface Specification}

Achieving interoperability among heterogeneous information sources ${ }^{2}$ requires common interfaces for all provided services. After the success of SQI [7], we decided to start a similar initiative towards the specification of these interfaces. The core services we consider include discovery, publishing and rating of knowledge resources as well as information source and user management.

\section{Knowledge Resource Discovery}

Efficient search for knowledge resources contained in repositories, P2P networks and Web 2.0 applications, requires a common search interface. There already exist a number of different standards for knowledge resource discovery with varying complexity [8]. Among them, the Simple Query Interface (SQI) is an outstanding solution which allows for session based synchronous and asynchronous querying of information sources, stateful and stateless communications and it is query language and result format independent. SQI has been our choice for knowledge resource discovery due to its simplicity and flexibility for different scenarios.

\section{Knowledge Resource Publishing}

A publishing service interface should enable a client to store knowledge resources and/or their metadata in an information source, be it locally, in an institutional repository or in a Web 2.0 application. In this way, the process is the same for any knowledge resource and information source, such that the user can perform this task without having to change the client application she is familiar with. Furthermore, in this way peers in a P2P network may obtain an additional possibility to publish their resources in an external repository in a persistent and standard manner such that these resources stay available after such peer goes offline.

The specification of the Simple Publishing Interface (SPI) is a collaborative effort among various European

\footnotetext{
2 With information sources we mean all kinds of information container e.g., repositories, $\mathrm{P} 2 \mathrm{P}$ networks and Web 2.0 applications.
}

projects (e.g., [1] [9] [10] [11]) and identifies and addresses all requirements of their scenarios.

\section{Information Source Management}

Since users may interact with different information sources (e.g., for querying or publishing of knowledge resources), an interface is required in order to retrieve description of these information sources (e.g., name and URL together with information on the supported services, languages and metadata formats) as well as to select the ones to be used in subsequent tasks. This information can be obtained from the Simple Information Source Management Interface (SISMI).

\section{User Management}

The Simple User Management Interface (SUMI) specifies several methods for retrieval and modification of user data. In addition, it manages user information required for accessing different information sources. This includes storage of the user $\log$ in data for the connected information sources as well as its use for the authentication purposes.

\section{Resource Rating}

Knowledge resources in an infrastructure like the one described above are not suitable for standard link analysis techniques like the ones used on the Web. Term frequency based ranking techniques are also not always applicable as knowledge resources do not necessarily contain textual information. A solution can be provided by enabling users to rate knowledge resources. Collaborative filtering techniques can then be used to rank the results. The Simple Resource Rating Interface (SRRI) provides methods for assigning such rating values to knowledge resources as well as for the retrieval of this information.

\section{Service Architecture \& Implementation}

Integration of heterogeneous information sources within a single infrastructure raises the challenge of interoperability, both on the technical and on the semantic level. Due to the limitations (both in metadata and query capabilities) of some of the information sources we are considering (especially the web systems) we started with a basic core set of metadata (a subset of Dublin Core and therefore of LOM) and rely on keyword based search. We implemented a 3-layered open source infrastructure which supports searching and publishing (storage, update and deletion) of knowledge resources, as well as other advanced services like e.g., user management and resource rating. All these services implement the interfaces described in the previous section and allow for knowledge resource sharing and management in both the local system and remotely (repositories and Web 2.0 applications). Our Service Oriented Architecture (Figure 1) relies on a 
registry in which information sources can be added dynamically.
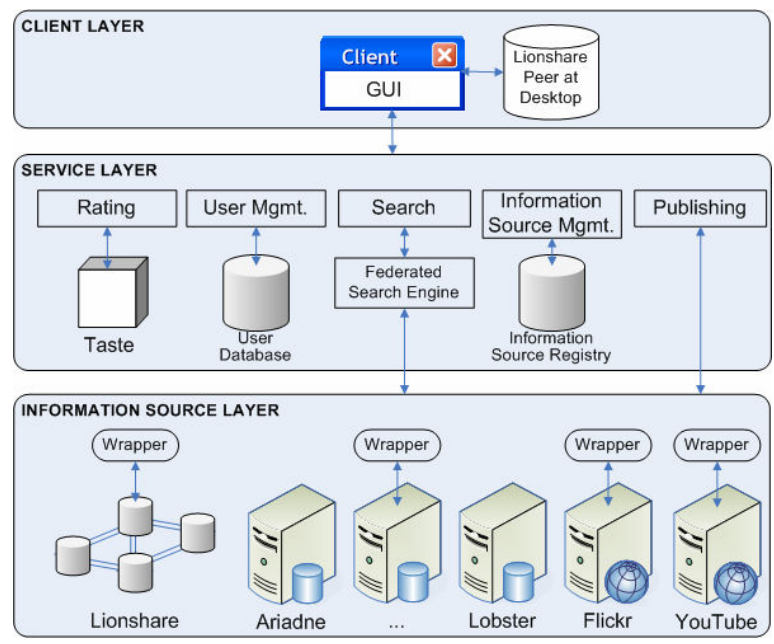

Figure 1: KRSM Architecture

\section{Information Source Layer}

The information source layer consists of the set of repositories, $\mathrm{P} 2 \mathrm{P}$ networks and Web 2.0 applications to be integrated in our infrastructure. Each information source must conform to the interfaces described above for each service provided, or implement a wrapper module for interface translation and metadata mapping.

\section{Service Layer}

The service layer provides search and publishing services as well as services for information source, session and user management. This layer contains the services that will be accessed and composed by client applications. Services in this layer may also make use of other services from the same layer.

\section{Client Layer}

The client layer provides a graphical user interface that connects the services and the user. We envision the use of both stand-alone and web-based applications. The former enables managing and sharing local knowledge resources via the local LionShare peer. The latter has the advantage that it may be accessed from anywhere without the need for any software installation. We support both scenarios though our implementation currently focuses on the first one.

\section{Related Work}

OAI-PMH [12] and CORDRA [13] rely on metadata harvesting. Our scenario does not require target information sources to disclose all their metadata. The IMS Digital Repository Interface specification [14] provides architectural examples and recommendations for query languages and messaging protocols but lacks a concrete binding of common repository services. The Open Knowledge Initiative (OKI) [15] provides a Java API to ease the development and coupling of components and offers a set of Open Service Interface Definitions (OSID). OSID represent complex application interfaces at a different level of abstraction than our architecture. We focus on simple and reliable interface definitions for communication among heterogeneous systems.

\section{Conclusions and Future Work}

Nowadays, learners require a more flexible approach for lifelong learning, making use of the large amount of knowledge resources available as well as collaborating with other users. This paper identifies the requirements a knowledge resource sharing and management infrastructure should address in order to integrate heterogeneous information sources and provide homogeneous search and publishing services. In addition, we have presented the work on the specification of simple but flexible and powerful interfaces, as well as the implementation of our open source service oriented architecture.

Although the results presented here are promising, there is still a lot of work to do. Further refinement and validation of the interfaces must be performed, extensions to allow more powerful query languages are being investigated and all our work needs to be validated as part of the overall infrastructure for the competence development and lifelong learning.

\section{References}

[1] TENCompetence, http://www.tencompetence.org

[2] Nejdl, Wolf, Qu, Decker, Sintek, Naeve, Nilsson, Palmer, and Risch. Edutella: A P2P networking infrastructure based on RDF. In $11^{\text {th }}$ International World Wide Web Conference, Hawaii, USA, June 2002.

[3] LionShare, http://lionshare.its.psu.edu/

[4] Brunkhorst and Olmedilla. "Interoperability for peer-topeer networks: Opening p2p to the rest of the world", ECTEL Conference, Heraklion, Greece, Oct. 2006.

[5] Flickr, http://www.flickr.com

[6] YouTube, http://www.youtube.com

[7] Van Assche, Duval, Massart, Olmedilla, Simon, Sobernig, Ternier and Wild. "Spinning interoperable applications for teaching \& learning using the simple query interface", Educational Technology \& Society, 9(2), 2006.

[8] IMS query services white paper, http://www.imsglobal.org/query/imsQueryServices.html.

[9] MACE, http://www.mace-project.eu

[10] MELT, http://info.melt-project.eu

[11] PROLEARN, http://www.prolearn-project.org/

[12] Sompel, Nelson, Lagoze, Warner, Line "Resource Harvesting within the OAI-PMH Framework", D-Lib Magazine, Vol. 10, Nr. 12, Dec. 2004

[13] CORDRA, http://cordra.net

[14] IMS, http://www.imsglobal.org/

[15] OKI, http://www.okiproject.org 